\title{
Properties of the background of EPIC-pn onboard XMM-Newton
}

\author{
H. Katayama ${ }^{1}$, I. Takahashi ${ }^{2}$, Y. Ikebe ${ }^{3}$, K. Matsushita ${ }^{3}$, and M. J. Freyberg ${ }^{3}$ \\ 1 Department of Earth and Space Science, Graduate School of Science, Osaka University, 1-1 Machikaneyama, Toyonaka, \\ 560-0043 Osaka, Japan \\ e-mail: hkatayam@ess.sci.osaka-u.ac.jp \\ 2 Department of Physics, University of Tokyo, 7-3-1 Hongo, Bunkyo-ku, 113-0033 Tokyo, Japan \\ 3 Max-Planck-Institut für Extraterrestrische Physik, Postfach 1312, 85741 Garching, Germany
}

Received 30 September 2002 / Accepted 29 July 2003

\begin{abstract}
We have investigated the background properties of EPIC-pn onboard XMM-Newton to establish the background subtraction method. Count rates of the background vary violently by two orders of magnitude at the maximum, while during the most quiet period, these are stable within $8 \%$ at a $1 \sigma$ level. The overall spectrum is dominated by particle events above $5 \mathrm{keV}$, and its spatial variation is also found. The long-term variation of the background is also investigated with CAL CLOSED data, where the filter wheel was in closed position with the internal calibration source illuminating the sensitive area. The average background count rate decreased by 20\% from March 2000 to January 2001, but it regained in February 2001. For the modeling of the background spectrum, we investigate relations between the $2.0-7.0 \mathrm{keV}$ count rate and some characteristic parameters. The 2.0-7.0 keV background count rate shows a good correlation with the count rate of events outside the field of view. This correlation is usable for the modeling of the background.
\end{abstract}

Key words. X-rays: general - instrumentation: detectors

\section{Introduction}

In X-ray data analysis, understanding properties of background in the data is crucial in order to extract actual source X-rays, in particular for extended objects with low surface brightness like galaxy clusters and supernova remnants (SNRs) having low signal to noise ratios. The most sophisticated high quality X-ray detectors currently used in X-ray observations in orbit are X-ray CCDs, which was for the first time onboard ASCA (1993-2000; Tanaka et al. 1994) and are currently utilized on XMM-Newton (Jansen et al. 2001) and Chandra (Weisskopf et al. 2000). The background of ASCA/SIS (Solidstate Imaging Spectrometer) is rather stable and its count rate correlates with the local cutoff rigidity value, indicating that the background is mainly induced by high energy particles, except at the South Atlantic Anomaly (SAA) (see e.g. Gendreau 1995a; Ueda 1996). On the other hand, the X-ray CCD cameras onboard XMM-Newton and Chandra exhibit violent variability as large as two orders of magnitudes (Markevitch 2001; Lumb 2001). The responsible difference between ASCA and the currently working two satellites is their orbits. ASCA took an almost circular orbit with an average

Send offprint requests to: K. Matsushita, e-mail: matsushita@rs.kagu.tus.ac.jp altitude of 520-620 km, while XMM-Newton and Chandra take an highly elliptical orbit with an apogee of about $100000 \mathrm{~km}$ and a perigee of about $10000 \mathrm{~km}$ and thus mostly fly outside the Earth's magnetosphere. The rapid variation is thought to be caused by protons scattered through the mirror system (often referred to as "soft protons"). In particular, protons in the energy of 100-200 keV degrade the performance of Chandra ACIS-I (Prigozhin et al. 2000). Lumb (2001) suggests that the rapid flux variation of those soft protons is caused by magnetic reconnections rather than solar flares.

XMM-Newton, with the largest effective area of the X-ray telescope and the high quantum efficiency of the CCD cameras, should be the best suitable observatory for low surface brightness objects. In this paper, we show the properties of the background of the EPIC-pn onboard XMM-Newton, following reports by Briel et al. (2001), Freyberg et al. (2002a,b), and Lumb (2001). We also study correlations between the background and some characteristic parameters to look for a possible method of the background modeling.

\section{Data and screening}

We used EPIC-pn data taken during the calibration and performance verification (Cal-PV) phase. We selected data sets taken in full frame mode with thin 1 filter, excluding data with 

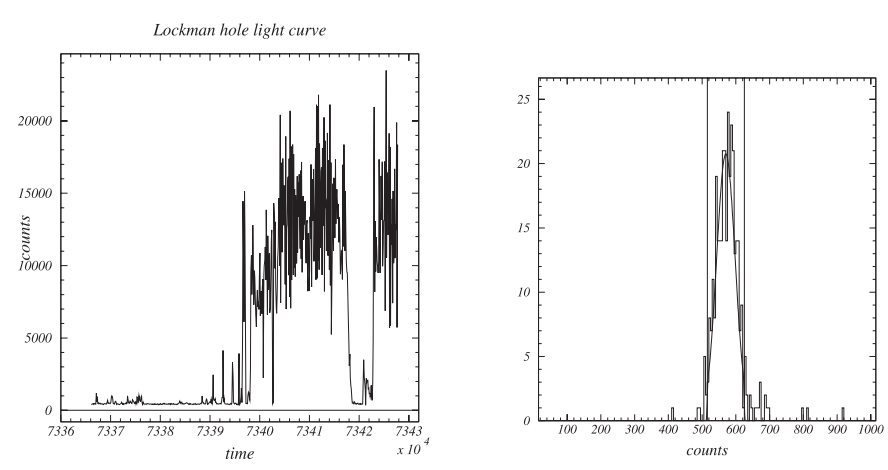

Fig. 1. (Left) Light curve of a Lockman Hole observation. (Right) Count rate histogram of the light curve. Two vertical lines are the thresholds of $\pm 2 \sigma$.
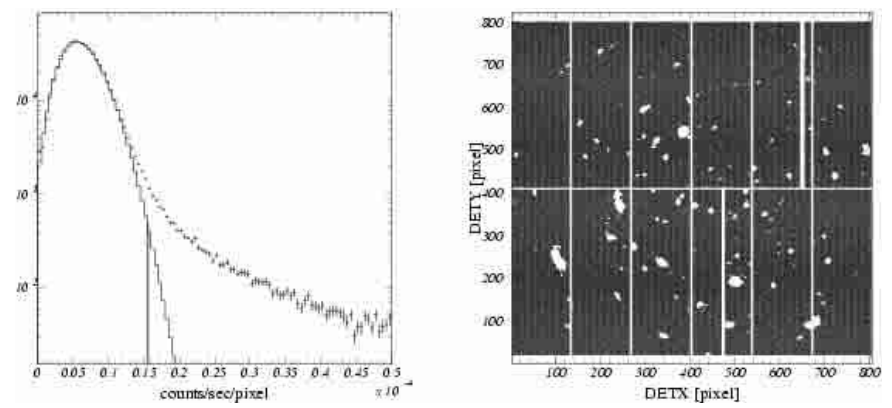

Fig. 2. (Left) Pixel-counts histogram. The histogram of count rate of each image bin from the smoothed image. Solid line represents the best fit of Poisson distribution. The vertical line is the threshold of 2.5 times the average. (Right) Image masked pixels exceed the threshold.

bright sources and observation time longer than $15 \mathrm{ks}$ to ensure enough count statistics. In addition to those data sets, we also use a data set taken with the filter wheel in the closed position (hereafter referred as CLOSED), which includes no contributions from photons or particles through the X-ray telescope. The data sets used in our analysis are summarized in Table 1.

We have selected single and double pattern events. Figure 1 shows a light curve from one of the Lockman hole observations. The light curve mainly consists of two characteristic components. One is the flaring component that is characterized by strong and rapid variability by two orders of magnitude at the maximum. The other part of the light curve shows rather stable count rates and ought to be used for scientific analysis. In order to exclude the flaring component, time periods where the count rate deviates from the mean value during the quiescent period by $\pm 2 \sigma$ are excluded. We should note that there are remaining low flux soft protons even after the flares are excluded. The fraction of such protons to the background is important for accurate background estimation. However the effect of low flux soft protons is still under investigation (Marty et al. 2002). Celestial sources and noisy CCD pixels are also excluded from the data in the following way. The image was binned with 40 sky pixels $\left(2^{\prime \prime}\right)$ and smoothed by a Gaussian of $\sigma=4^{\prime \prime}$. From the smoothed image made, we then made a pixel-counts histogram, which is the histogram of the count rate of each image bin (Fig. 2). The pixel-counts histogram was fitted with a Poisson distribution. The pixels with count rates

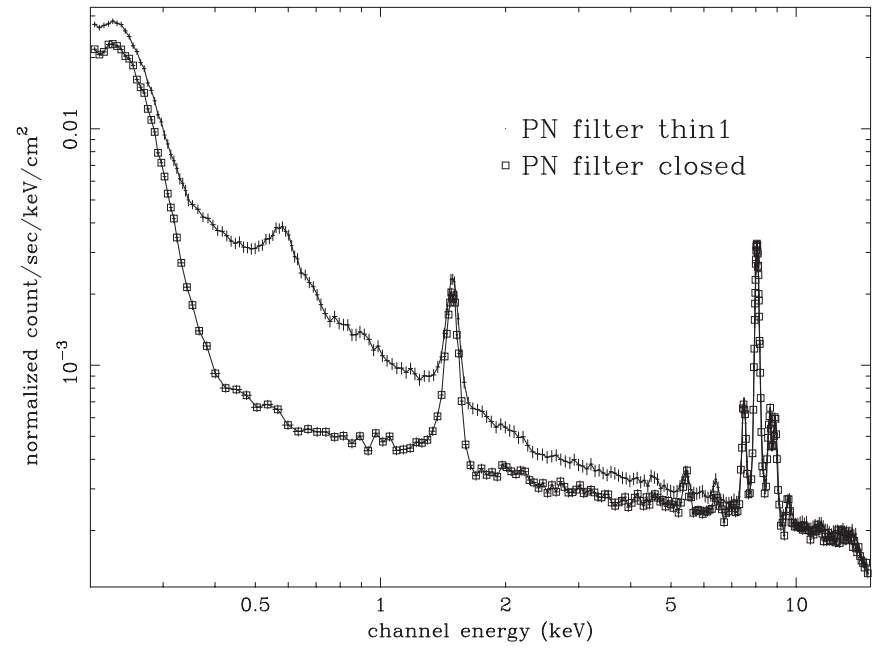

Fig. 3. Average (dot) and internal (open square) background spectra of the EPIC-pn. The prominent line features are Al-K (1.49 keV), Ni-K (7.48 keV), Cu-K (8.05,8.91 keV), and Zn-K (8.64, $9.57 \mathrm{keV})$ fluorescence lines.

exceeding 2.5 times the average value were excluded as a source or noisy pixels. After eliminating those high count pixels, we again made a light curve and performed the temporal screening as described above. The average exposure time remaining after the screening is about $19 \mathrm{ks}$. The sample may represent typical observations. In the following sections, we investigate stability of the energy spectrum and the spatial distribution of the background.

\section{Spectrum of EPIC-pn background}

Figure 3 shows the average background spectrum of EPICpn obtained from observations of all the thin filter data listed in Table 1. Compared with the energy spectrum from the CLOSED data, which is overlaid in the same figure, the background spectrum is found to be dominated by the particle origin events above $5 \mathrm{keV}$, while, below $5 \mathrm{keV}$, the cosmic X-ray background $(\mathrm{CXB})$ is the major contribution. Below $0.3 \mathrm{keV}$, another non-X-ray noise emerges. The prominent line features are Al-K (1.49 keV), Ni-K (7.48 keV), Cu-K (8.05, $8.91 \mathrm{keV})$, and $\mathrm{Zn}-\mathrm{K}(8.64,9.57 \mathrm{keV})$ lines (Freyberg et al. 2002a). The excess emission at $0.6 \mathrm{keV}$ is very likely due to characteristic emission lines from OVII and OVIII that associated with the local bubble (e.g. Tanaka \& Bleeker 1977; Gendreau et al. 1995b)

We next accumulated energy spectrum from each data set and obtained its ratio to the average spectrum, which are shown in the left panel of Fig. 4. The variations of count rate in each energy band are quantified with the standard deviation at a $1 \sigma$ and illustrated in the right panel of Fig. 4 . In the $0.5-12 \mathrm{keV}$, it is almost constant at $\sim 8 \%$, while below $0.4 \mathrm{keV}$ it increases to $\sim 14 \%$. The variations at $\mathrm{Al}$ and $\mathrm{Cu}$ lines are significantly smaller than those of continuum. These lines come from the electronic board below the CCD and do not affected by the soft protons, since those which can be scattered through the mirror system can not penetrate the CCD. Therefore, high energy particles which induce these lines may have a smaller variability than the soft protons. 
Table 1. PN data summary.

\begin{tabular}{|c|c|c|c|c|c|c|}
\hline \multicolumn{7}{|c|}{ Filter Thin } \\
\hline Observation ID & $\begin{array}{l}\text { Date of } \\
\text { observation }\end{array}$ & $\begin{array}{l}\text { Orbital }^{a} \\
\text { phase } \\
{[\mathrm{h}]}\end{array}$ & $\begin{array}{l}\text { Exposure }^{b} \\
{[\mathrm{ks}]}\end{array}$ & $\begin{array}{l}\text { ERMS }^{c} \\
\text { count } \\
\text { NHE0/NHE1 }\end{array}$ & Object & Symbols $^{d}$ \\
\hline 0063_0123100201_PNS001 & $2000-04-12$ & 13.8 & 11.4 & $15.9 / 6.2$ & MS0737.9+7441 & closed circle \\
\hline 0070_0123700101_PNS003 & $2000-04-27$ & 22.4 & 27.7 & $16.7 / 6.5$ & Lockman Hole & closed square \\
\hline 0071_0123700201_PNS003 & $2000-04-29$ & 22.4 & 21.0 & $16.7 / 6.6$ & Lockman Hole & closed triangle \\
\hline 0073_0123700401_PNS003 & $2000-05-02$ & 13.8 & 10.0 & $16.9 / 6.5$ & Lockman Hole & open circle \\
\hline 0078_0124100101_PNS003 & $2000-05-13$ & 22.3 & 21.4 & $16.5 / 6.4$ & RXJ0720.4-3125 & open square \\
\hline 0081_0123701001_PNS003 & 2000-05-19 & 25.5 & 17.6 & $--^{e}$ & Lockman Hole & open triangle \\
\hline 0082_0124900101_PNS003 & $2000-05-21$ & 22.3 & 17.7 & $16.4 / 6.3$ & MS1229.2+6430 & open diamond \\
\hline 0086_0125300101_PNS013 & $2000-05-28$ & 7.8 & 26.2 & $14.9 / 5.8$ & $\mathrm{~J} 104433.04-012502.2$ & closed asterisk \\
\hline 0181_0098810101_PNS003 & 2000-12-04 & 15.9 & 18.5 & 21.8/7.6 & WW Hor & open asterisk \\
\hline \multicolumn{7}{|c|}{ CLOSED } \\
\hline Observation ID & $\begin{array}{l}\text { Date of } \\
\text { observation }\end{array}$ & $\begin{array}{l}\text { Orbital }^{a} \\
\text { phase } \\
{[\mathrm{h}]}\end{array}$ & $\begin{array}{l}\text { Exposure }^{b} \\
{[\mathrm{ks}]}\end{array}$ & $\begin{array}{l}\text { ERMS }^{c} \\
\text { count } \\
\text { NHE0/NHE1 }\end{array}$ & & \\
\hline 0059_0122320701_PNS003 & $2000-04-05$ & 25.5 & 42.3 & $129.5 / 17.1$ & & \\
\hline
\end{tabular}

${ }^{a}$ Hours after perigee exit.

${ }^{b}$ After the screening of the flaring component.

${ }^{c}$ Count rate of EPIC radiation monitor (high energy part).

${ }^{d}$ Symbols in Fig. 4 (left), 5, 11, 12, and 13.

${ }^{e}$ No data.
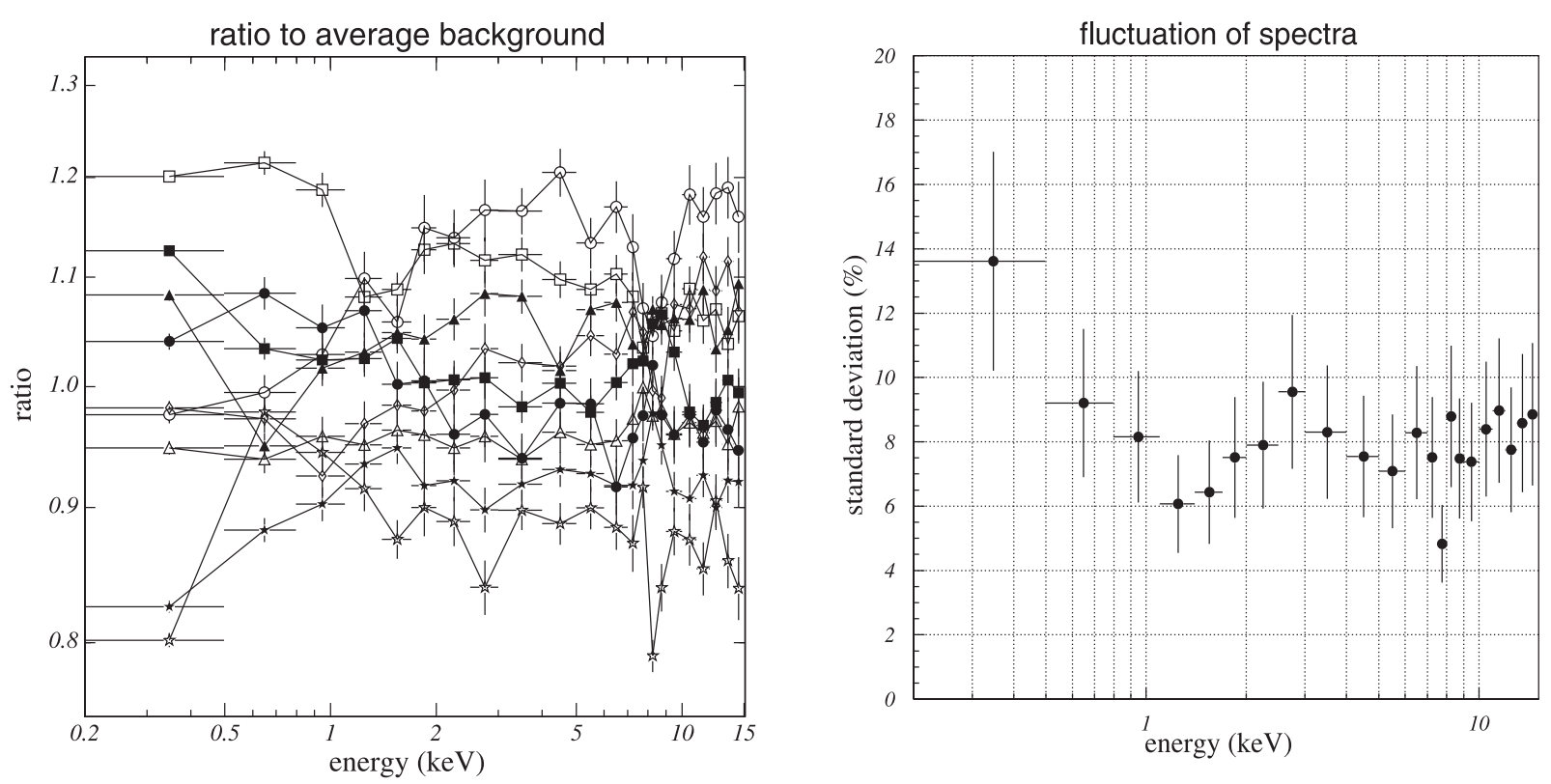

Fig. 4. (Left) Ratios of individual background spectrum to the average. The meaning of the symbols are summarized in Table 1. (Right) Standard deviation of background spectra in each energy band at a $1 \sigma$ level.

In order to investigate the stability of the shape of the background spectrum, we divided the spectrum into seven energy bands $(0.2-0.4 \mathrm{keV}, 0.4-1.3 \mathrm{keV}, 1.3-1.7 \mathrm{keV}, 1.7-4.0 \mathrm{keV}$, $4.0-7.2 \mathrm{keV}, 7.2-9.2 \mathrm{keV}$, and 9.2-12.0 keV) and derived the ratios between different energy bands. Figure 5 shows the relations of count rates between $4.0-7.2 \mathrm{keV}$ and the other six energy bands. In the $1.3-12.0 \mathrm{keV}$ band, the shape of the background spectrum is stable within $5 \%$. Below $1.3 \mathrm{keV}$, no good correlation is seen. In the $0.4-1.3 \mathrm{keV}$ band, this is probably due to variation of the CXB component in the soft energy region. No correlation seen between the $0.2-0.4 \mathrm{keV}$ and 4.0 $7.2 \mathrm{keV}$ indicates that the non-X-ray noise that dominates the $0.2-0.4 \mathrm{keV}$ count rate vary independently from the CXB and the particle origin events. 

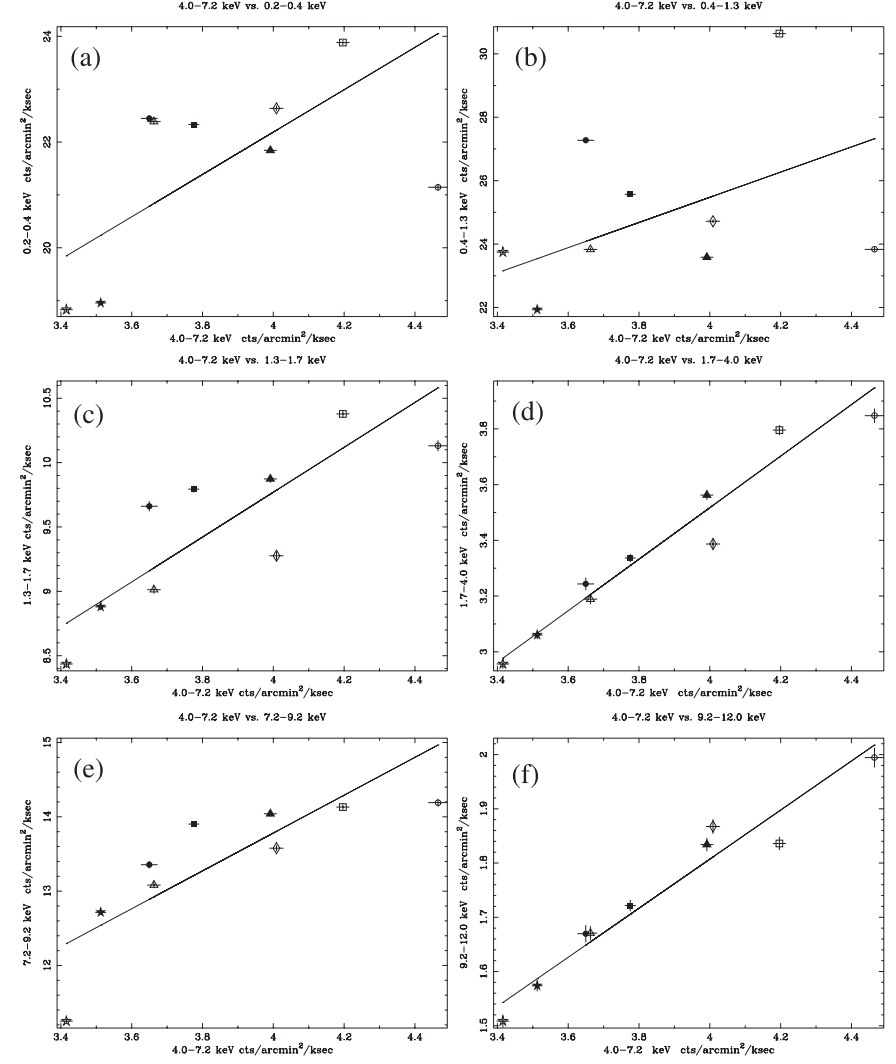

Fig. 5. Relations of background count rates between 4.0-7.2 keV and other six energy bands; a) $0.2-0.4 \mathrm{keV}$, b) $0.4-1.3 \mathrm{keV}$, c) $1.3-1.7 \mathrm{keV}$, d) $1.7-4.0 \mathrm{keV}$, e) $7.2-9.2 \mathrm{keV}$, and f) $9.2-12.0 \mathrm{keV}$. The meaning of the symbols are summarized in Table 1. The solid lines correspond to the best fit regression lines.

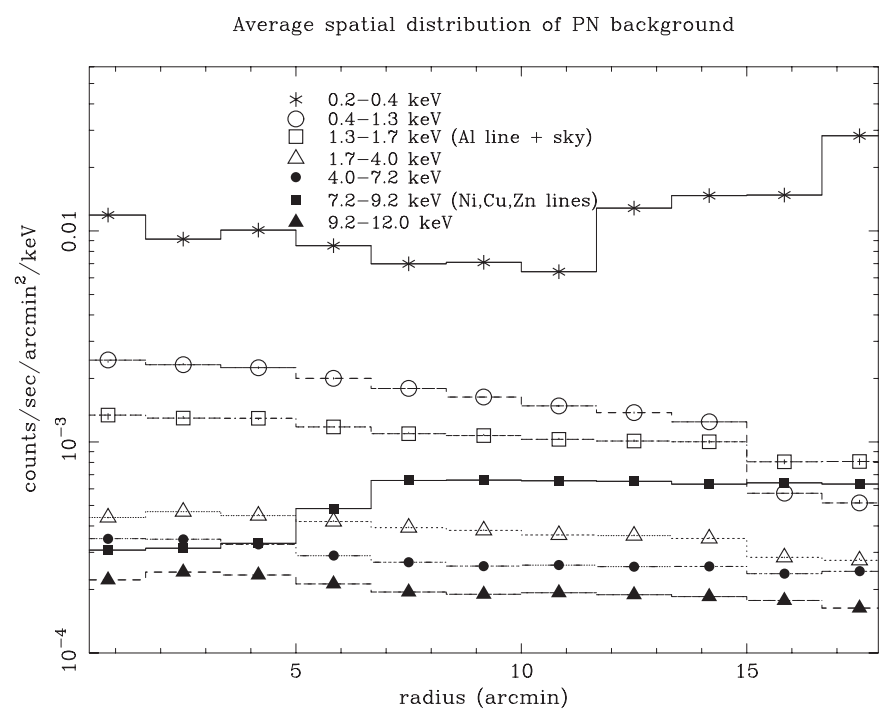

Fig. 6. Radial count rate profile of EPIC-pn background.

\section{Spatial distribution of background}

Figure 6 displays the radial count rate profile of the backgrounds in different energy bands. In the $0.2-0.4 \mathrm{keV}$ band profile, the count rate increases beyond $\sim 12$ arcmin, which caused by a concentration of the non-X-ray noise around the readout node. In the $0.4-4.0 \mathrm{keV}$ band where $\mathrm{CXB}$ dominates, the

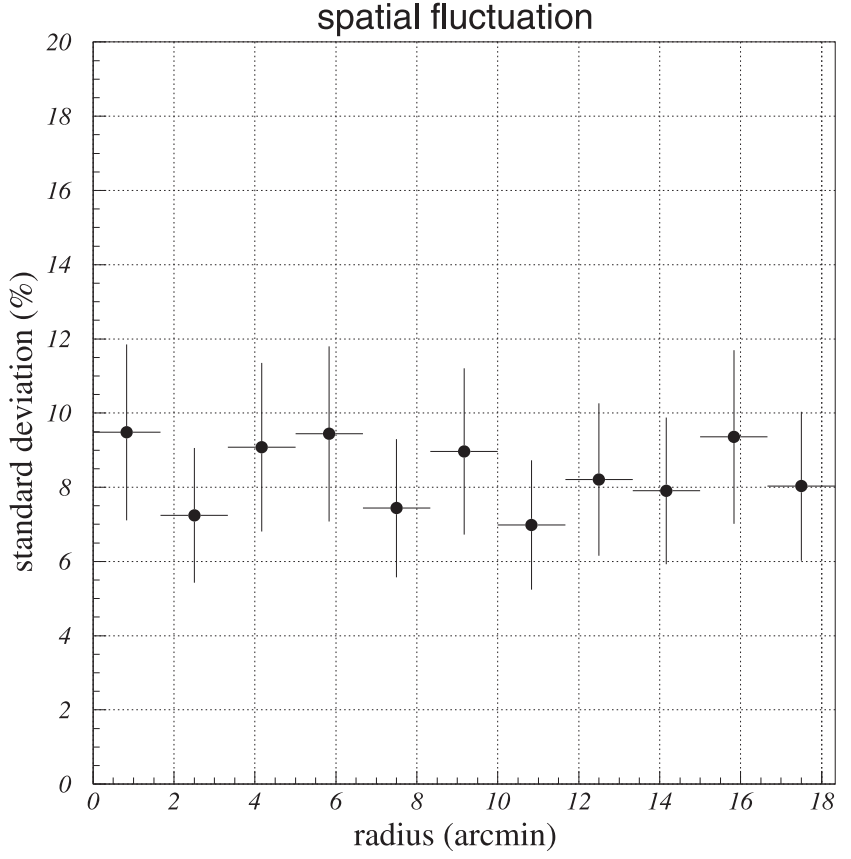

Fig. 7. Standard deviation of the Radial profile of EPIC-pn background.

profiles show the telescope vignetting effect. A sudden drop at 15 arcmin radius corresponds to the edge of the field of view (FOV), outside which no sky X-rays are supposed to be detected. The 7.2-9.0 keV band is dominated by $\mathrm{Cu}$ line, which is originated from the electronics circuit board placed beneath the CCD except the central about 6 arcmin region (see Freyberg et al. 2002b) In the highest energy band, 9.2-12.0 keV, the count rate profile is almost flat with some excess in the central $\sim 6$ arcmin region, which may correspond to the structure of the electronics circuit board. We summarized characteristics of all background components in Table 2 .

As for the energy spectrum, we examined the stability of the radial count rate profile. Figure 7 shows the $1 \sigma$ standard deviation in the $2.0-7.0 \mathrm{keV}$ band, which is virtually constant over the detector. Figure 8 shows the correlations of count rates between outside of FOV and inside of FOV for different energy bands $(0.2-0.4 \mathrm{keV}, 0.4-1.7 \mathrm{keV}, 1.7-7.2 \mathrm{keV}$, and 7.2$12.0 \mathrm{keV})$. Open circles, filled circles, and filled squares indicate the count rate in the $r=0-5^{\prime}, 5-10^{\prime}$, and $10-15^{\prime}$, respectively. Each count rate is normalized by the average count rate. From these plots, the shape of the radial count rate profiles found to be unchanged within $4 \%$ for the $1.7-7.2 \mathrm{keV}$ band and $10 \%$ for the $7.2-12.0 \mathrm{keV}$ band. No good correlation is seen in the $0.2-0.4 \mathrm{keV}$ band and the $0.4-1.7 \mathrm{keV}$ band, which is very likely due to the variation of the non-X-ray noise and the $\mathrm{CXB}$, respectively.

\section{Long-term variation of the background}

We investigated long-term variation of the background count rate. For the study, we used CAL CLOSED data, which is taken during the CCD is exposed by the calibration source mounted on the filter wheel. This operation is performed every orbit 
Table 2. Characteristics of all background components.

\begin{tabular}{lll}
\hline \hline Band $(\mathrm{keV})$ & Vignetted & Note \\
\hline $0.2-0.4$ & no & non-X-ray noise around the readout node \\
$0.4-1.3$ & yes & $\mathrm{CXB}$ dominant \\
$1.3-1.7$ & yes & $\mathrm{Al} \mathrm{K}$ line \\
$1.7-4.0$ & yes & $\mathrm{CXB}+$ Internal background \\
$4.0-7.2$ & yes & $\mathrm{CXB}+$ Internal background \\
$7.2-9.2$ & no & $\mathrm{Ni}, \mathrm{Cu}$, and Zn lines, Structure of electronics circuit board \\
$9.2-12.0$ & no & Internal background dominant \\
\hline
\end{tabular}

Correlation $0.2-0.4 \mathrm{keV}$

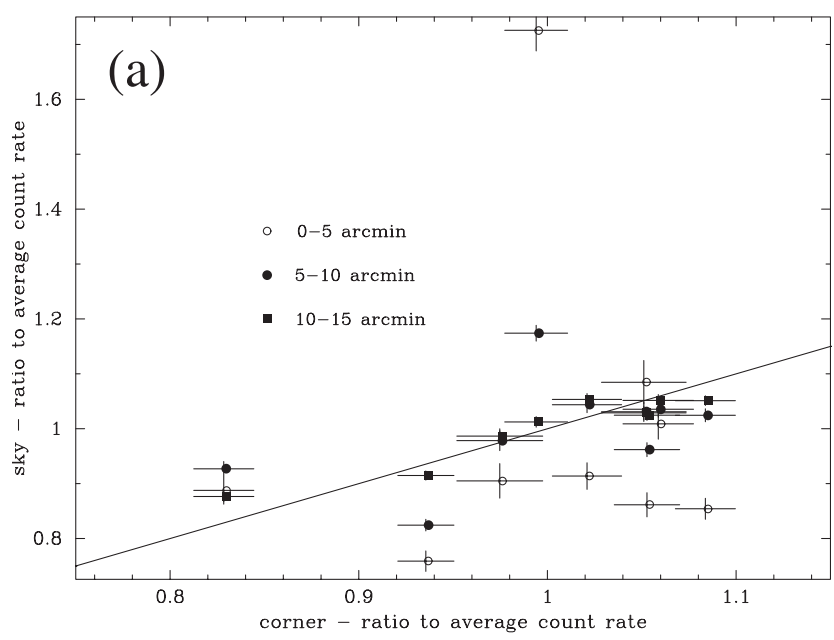

Correlation $1.7-7.2 \mathrm{keV}$

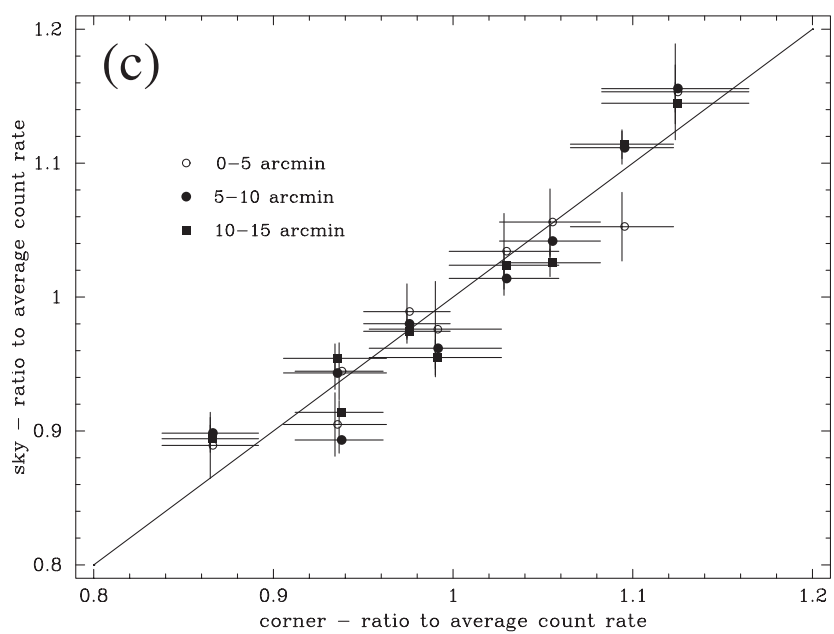

Correlation $0.4-1.7 \mathrm{keV}$

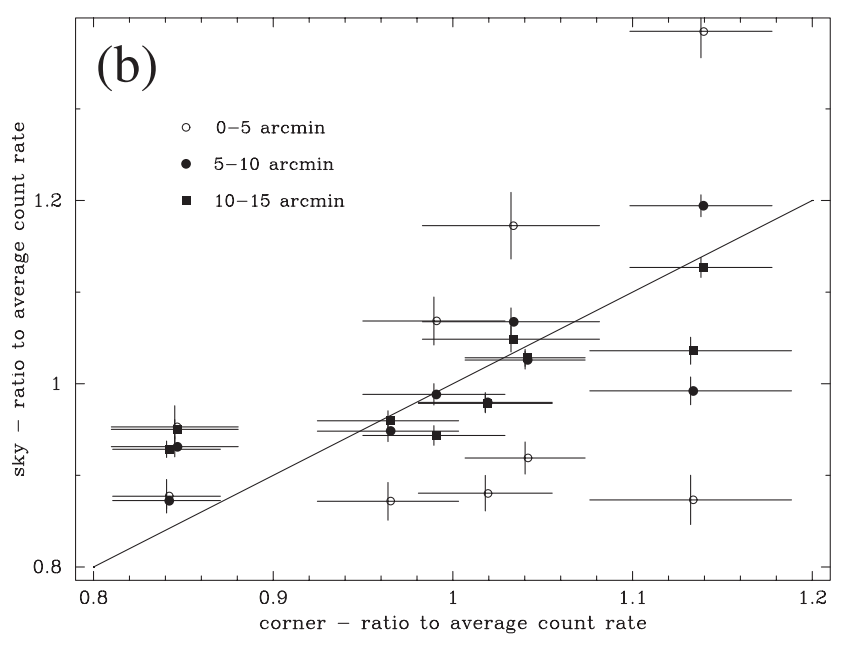

Correlation $7.2-12.0 \mathrm{keV}$

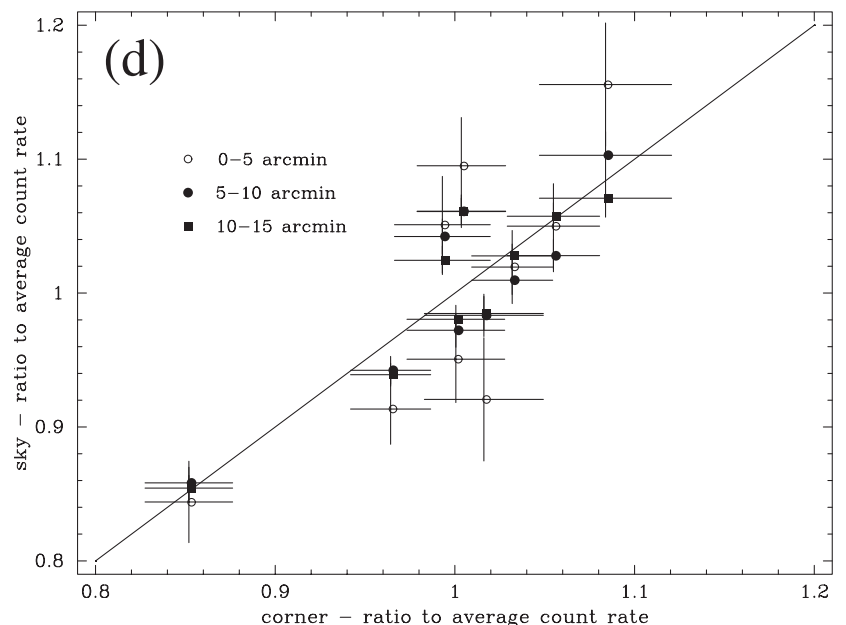

Fig. 8. Correlations of count rate between the region of outside of FOV (corner) and inside of FOV (sky) in different energy bands; a) 0.2$0.4 \mathrm{keV}$, b) $0.4-1.7 \mathrm{keV}$, c) $1.7-7.2 \mathrm{keV}$, and d) $7.2-12.0 \mathrm{keV}$. Each count rate is normalized by the average count rate. Open circles, filled circles, and filled squares indicate the count rate in the $r=0-5^{\prime}, 5-10^{\prime}$, and $10-15^{\prime}$, respectively. The solid lines correspond to the best fit regression lines.

during the satellite orbit is the closest to the Earth and no observation is possible due to high flux of low energy electrons. In Table 3, the CAL CLOSED observations we used are listed.

The data are free from sky X-rays and soft protons and could be used for monitoring particle background. Figure 9 shows the $5.0-13.0 \mathrm{keV}$ spectra taken from one of CAL CLOSED data compared with the Lockman hole data and the CLOSED data. The contribution from the calibration source is found only below $7.2 \mathrm{keV}$. Therefore, the $7.2-13.0 \mathrm{keV}$ band of the CAL CLOSED data can be usable for monitoring the count rate of the particle origin events.

Figure 10 displays the long-term light curve in the 7.88.3 $\mathrm{keV}$ and 10.0-13.0 keV band derived from the CAL CLOSED data from March 2000 to October 2001. The average background count rate decreased by $20 \%$ from March 2000 to January 2001, while, it regained in February 2001. Before 


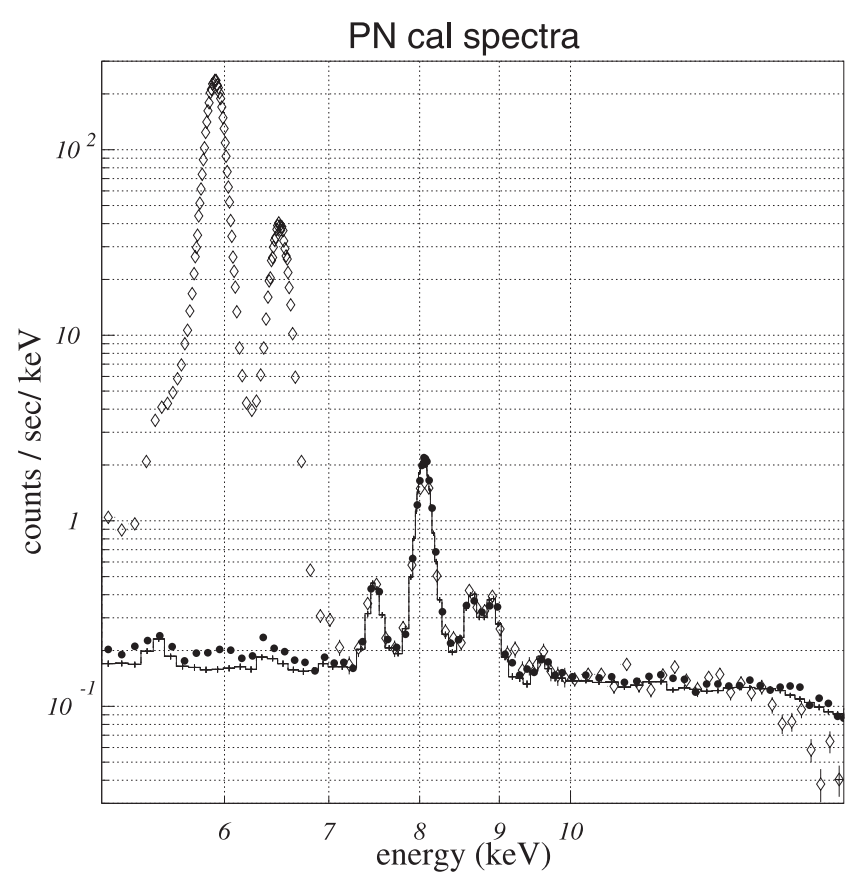

Fig. 9. Spectra of CAL CLOSED (open diamond), Lockman hole (filled circle), and CLOSED (solid line) data.

January 2001, the same trend has been found in the Chandra ACIS background (see Fig. 9 on Markevitch 2001). The change at February 2001 corresponds to a change of the operation; after February 2001, the CAL CLOSED data are taken at positions with high radiation, when no scientific observations can be performed. Note that most of the data we used in Sects. 3 and 4 are taken from 103rd to 149th day, and the long-term variations do not affect the results.

\section{Possible methods of the background modeling}

Although, the count rate of the background in quiescent period is rather stable (within $8 \%$ for $1 \sigma$ ), a data set may have different count rate as large as $50 \%$ or more from the average value as seen in the long-term light curve. Therefore, applying the average background may not work generally.

In this section, we look for a method to predict the background count rate for an observation, that should work for the data where the source emission covers the entire FOV, like an observation of a nearby cluster. As a parameter for monitoring the background count rate, we here consider the following three parameters: (1) number of discarded columns, (2) count rate of 10.0-13.0 keV, and (3) count rate of outside of FOV.

\subsection{Number of discarded columns}

In the data processing of EPIC-pn, the column that detects an event above upper energy threshold and its neighbor columns are removed from the data as a background (Appendix of Freyberg et al. 2002b). This is called minimum ionizing particle (MIP) rejection. As most of these MIP events are particle events, the number of columns that are discarded by the process is expected to be related to the remaining background

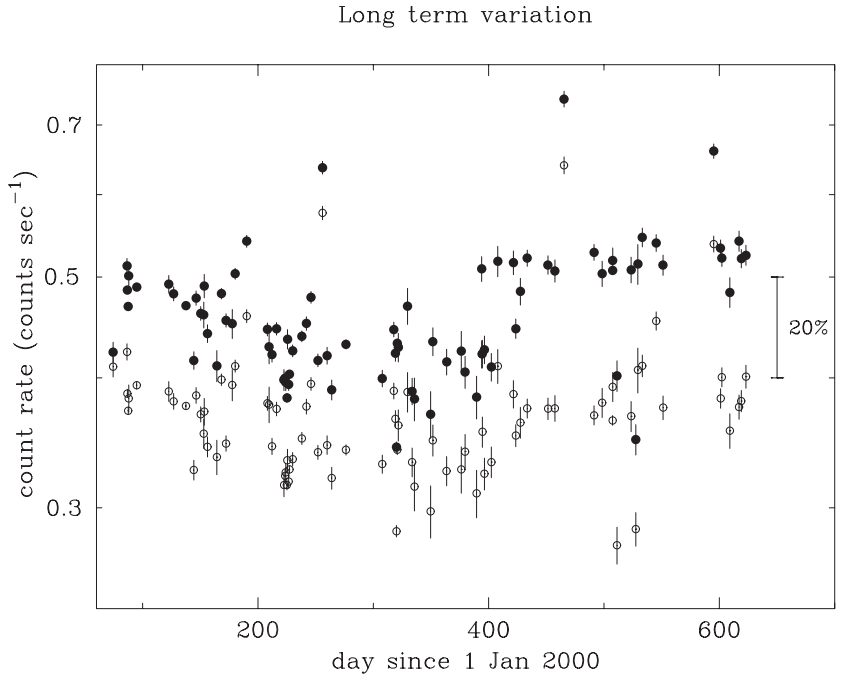

Fig. 10. Long-term light curve in the $7.8-8.3 \mathrm{keV}$ (filled circle) and 10.0-13.0 keV (open circle) band derived from the CAL CLOSED data.

count rate. However, as shown in Fig. 11, there is no clear correlation between the $2.0-7.0 \mathrm{keV}$ count rates and the number of discarded columns. The correlation coefficient for this data set is 0.544 .

The MIP events are generated by high energy particles which pass through or stop in the CCD with losing its energy. On the other hand, the $2.0-7.0 \mathrm{keV}$ background, in particular the continuum, is attributed to a smaller number of particles that have a certain configuration of the energy and the incident direction. Therefore, the $2.0-7.0 \mathrm{keV}$ background count rate would fluctuate largely by the temporal change of the particle energy spectrum. As shown in the low-gain observation of EPIC-pn, the spectrum of low-energy particles $(E<250 \mathrm{keV})$ changes below $50 \mathrm{keV}$ (see Fig. 9 in Strüder et al. 2001). This indicates the population of high energy particles is not simply consist of single component. Further investigation with the low gain observation data would be helpful to understand the origin of the background.

\subsection{Count rate of $10.0-13.0 \mathrm{keV}$}

For many sources, X-ray flux above $10.0 \mathrm{keV}$ are negligibly small compared to the background. Thus, the count rate above $10.0 \mathrm{keV}$ could be used for modeling the background.

In Fig. 12, the background count rates in $10.0-13.0 \mathrm{keV}$ are plotted against those in $2.0-7.0 \mathrm{keV}$, showing a good correlation. The correlation coefficient for these data is 0.929 , and the scatter is less than $8 \%$.

\subsection{Count rate of outside of FOV}

Events detected in the outside of FOV are mostly originated from high energy particles and are expected to be a good monitor of the background in the FOV. Figure 13 displays the 2.0$7.0 \mathrm{keV}$ count rate detected outside of FOV vs. that in FOV. It shows a good correlation. The correlation coefficient is 0.983 , and the scatter is less than $3 \%$. Therefore, the count rate of 
Table 3. CAL CLOSED data summary.

\begin{tabular}{|c|c|c|c|c|c|}
\hline Observation ID & $\begin{array}{l}\text { Date of } \\
\text { observation }\end{array}$ & $\begin{array}{l}\text { Exposure }^{a} \\
{[\mathrm{ks}]}\end{array}$ & "Observation ID & $\begin{array}{l}\text { Date of } \\
\text { observation }\end{array}$ & $\begin{array}{l}\text { Exposure }^{a} \\
{[\mathrm{ks}]}\end{array}$ \\
\hline 0049_0094810801_PNS001 & $2000-03-15$ & 3.7 & 0171_0099280601_PNS010 & $2000-11-15$ & 5.4 \\
\hline 0055_0122310101_PNS003 & 2000-03-27 & 4.6 & 0172_0132530401_PNU032 & 2000-11-16 & 12.6 \\
\hline 0055_0122310201_PNS003 & $2000-03-27$ & 17.7 & 0172_0132530501_PNS001 & $2000-11-16$ & 15.6 \\
\hline 0055_0122310301_PNS003 & $2000-03-28$ & 23.8 & 0173_0106660301_PNS003 & 2000-11-17 & 1.6 \\
\hline 0055_0122310401_PNS003 & $2000-03-28$ & 2.2 & 0177_0109990501_PNS005 & $2000-11-25$ & 0.8 \\
\hline 0059_0122320101_PNS003 & 2000-04-04 & 21.7 & 0179_0112910401_PNS001 & $2000-11-29$ & 1.7 \\
\hline 0073_9999990071_PNU002 & 2000-05-02 & 3.4 & 0180_0109130901_PNS001 & $2000-12-01$ & 1.7 \\
\hline 0075_0124300101_PNS003 & $2000-05-06$ & 5.1 & 0187_0113050901_PNU002 & $2000-12-15$ & 4.0 \\
\hline 0080_0124700101_PNS003 & $2000-05-17$ & 35.1 & 0188_0112650601_PNS003 & $2000-12-17$ & 1.8 \\
\hline 0084_0125110101_PNU002 & $2000-05-24$ & 4.3 & 0194_0110011701_PNS005 & $2000-12-29$ & 1.7 \\
\hline 0085_0125120101_PNS005 & $2000-05-26$ & 5.4 & 0200_9020000004_PNS003 & 2001-01-11 & 1.5 \\
\hline 0087_0124930101_PNS003 & $2000-05-30$ & 6.3 & 0202_0099010201_PNS003 & 2001-01-14 & 1.2 \\
\hline 0088_0125320301_PNS010 & 2000-06-02 & 1.6 & 0207_0067190301_PNS003 & 2001-01-24 & 0.9 \\
\hline 0088_0125130101_PNU007 & 2000-06-02 & 1.8 & 0209_9020900002_PNS003 & 2001-01-28 & 1.5 \\
\hline 0090_0125920201_PNS008 & 2000-06-05 & 3.9 & 0209_9020900003_PNS003 & 2001-01-29 & 1.5 \\
\hline 0094_0126700101_PNU002 & 2000-06-13 & 1.7 & 0210_9021000002_PNS003 & 2001-01-31 & 1.5 \\
\hline 0096_0006810201_PNS003 & $2000-06-17$ & 12.4 & 0213_9021300003_PNS003 & 2001-02-06 & 1.5 \\
\hline 0098_0124711801_PNS001 & $2000-06-21$ & 6.1 & 0216_9021600002_PNS003 & 2001-02-11 & 1.5 \\
\hline 0100_0097820101_PNS013 & $2000-06-26$ & 1.3 & 0223_0022140501_PNS004 & $2001-02-25$ & 1.7 \\
\hline 0102_0096210101_PNS003 & 2000-06-29 & 9.5 & 0224_0093620801_PNU003 & 2001-02-27 & 2.9 \\
\hline 0107_0112600601_PNS010 & 2000-07-09 & 6.0 & 0226_0021750801_PNS006 & 2001-03-03 & 1.5 \\
\hline 0116_0097610901_PNS005 & $2000-07-27$ & 7.0 & 0229_0001730401_PNU002 & 2001-03-09 & 3.5 \\
\hline 0116_0102040901_PNS003 & $2000-07-28$ & 1.4 & 0238_0061540201_PNS003 & 2001-03-27 & 2.8 \\
\hline 0118_0110890601_PNS001 & $2000-07-31$ & 5.2 & 0241_0109990601_PNS005 & 2001-04-02 & 2.3 \\
\hline 0120_0102641801_PNS001 & 2000-08-04 & 7.2 & 0245_0107460101_PNS003 & 2001-04-10 & 2.6 \\
\hline 0123_0128720301_PNS001 & $2000-08-10$ & 2.8 & 0258_0112290401_PNS003 & 2001-05-06 & 3.5 \\
\hline 0123_0128720501_PNS001 & $2000-08-11$ & 8.4 & 0261_9026100003_PNS003 & 2001-05-13 & 1.5 \\
\hline 0124_0128720601_PNS001 & $2000-08-12$ & 5.1 & 0266_0136750301_PNU002 & 2001-05-22 & 14.1 \\
\hline 0124_0128720901_PNS001 & $2000-08-13$ & 16.7 & 0266_9026600001_PNS003 & 2001-05-22 & 1.5 \\
\hline 0124_0128721001_PNS001 & $2000-08-13$ & 3.1 & 0268_0093641301_PNS003 & 2001-05-26 & 3.8 \\
\hline 0125_0110010101_PNU002 & $2000-08-14$ & 8.6 & 0274_9027400002_PNS003 & 2001-06-07 & 1.5 \\
\hline 0125_0110010101_PNU015 & $2000-08-15$ & 31.9 & 0276_9027600002_PNS003 & 2001-06-11 & 1.5 \\
\hline 0127_0112260601_PNS003 & $2000-08-18$ & 8.2 & 0277_0136550201_PNS003 & 2001-06-13 & 1.0 \\
\hline 0131_0129121101_PNS011 & $2000-08-26$ & 9.3 & 0279_0047540701_PNS003 & 2001-06-17 & 2.5 \\
\hline 0133_0103861901_PNS001 & $2000-08-29$ & 5.9 & 0285_0109270501_PNS003 & 2001-06-29 & 3.8 \\
\hline 0135_0101440901_PNS003 & 2000-09-02 & 7.3 & 0288_0032141001_PNU002 & 2001-07-05 & 2.3 \\
\hline 0138_0101441201_PNS003 & 2000-09-08 & 7.3 & 0310_0134540501_PNS005 & 2001-08-18 & 3.8 \\
\hline 0140_0129340401_PNS001 & 2000-09-12 & 4.6 & 0313_0111070301_PNS005 & 2001-08-24 & 3.4 \\
\hline 0142_0104660201_PNS003 & $2000-09-16$ & 4.2 & 0313_0110012201_PNU002 & 2001-08-25 & 3.6 \\
\hline 0144_0105261901_PNS003 & 2000-09-20 & 3.2 & 0317_0089940701_PNS003 & 2001-09-01 & 2.1 \\
\hline 0150_0105262301_PNS003 & $2000-10-03$ & 13.4 & 0321_0109490801_PNS005 & 2001-09-09 & 2.1 \\
\hline 0166_0132121901_PNS001 & $2000-11-03$ & 5.2 & 0322_0111520101_PNS013 & 2001-09-11 & 2.8 \\
\hline 0171_0099280501_PNS010 & 2000-11-13 & 6.8 & 0324_0112660201_PNS005 & 2001-09-15 & 2.2 \\
\hline
\end{tabular}

${ }^{a}$ After the screening of the flaring component.

outside of FOV (hereafter COF) shows the best correlation among the three parameters we examined here. Note that although the out-of-time events (Strüder et al. 2001) that contaminates the outside of FOV have not been subtracted, $30 \%$ count rate fluctuation inside FOV causes the count rate variation in the outside FOV by at most $2 \%$.

The correlation should be used to predict background of an observation. A predicted background of an observation can be given from the average background scaled to have the same $\mathrm{COF}$ as that of the observation. In order to examine the efficacy, we renormalized each spectrum in Fig. 4 so that each COF in the $2.0-7.0 \mathrm{keV}$ band becomes the same as that of the average background, and calculated the variations (standard deviations of $1 \sigma$ ), which is shown in Fig. 14. The variation of the pn background spectra decreases from the original scatter of $8 \%$ to $2 \%$ in the $2.0-7.0 \mathrm{keV}$, while the soft energy band below $\sim 1 \mathrm{keV}$ do not change. This is because the COF represents only the flux of the high energy particle events. The variation in the 7.0-9.0 keV band where characteristic emission lines are detected was not improved as much as the $2.0-7.0 \mathrm{keV}$ band, either. This corresponds to the scatter seen in Fig. 5e. The radial profile of the standard deviation in the $2.0-7.0 \mathrm{keV}$ shows 


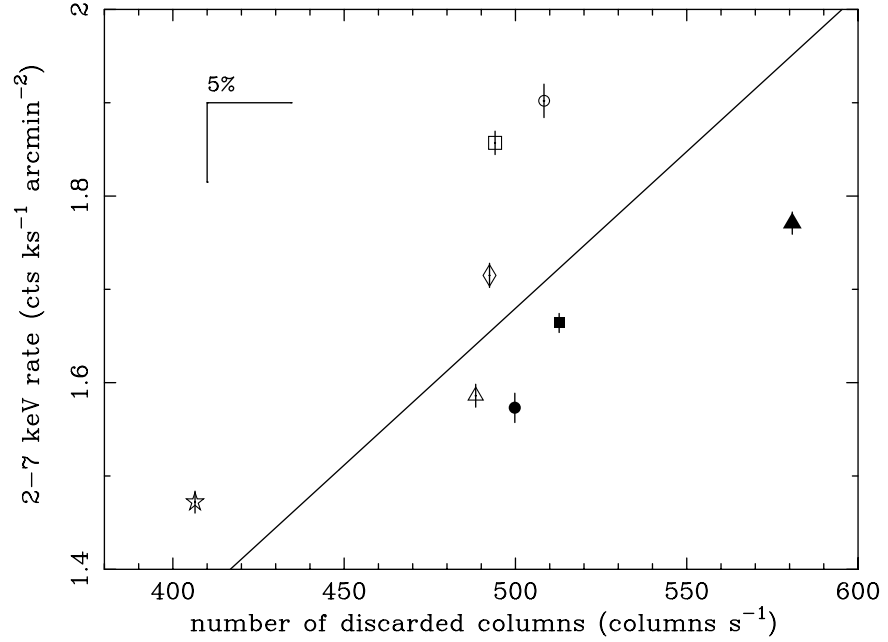

Fig. 11. Discarded column vs. $2.0-7.0 \mathrm{keV}$ background count rate. The correlation coefficient is 0.544 . The meaning of the symbols are summarized in Table 1. The solid line corresponds to the best fit regression line.

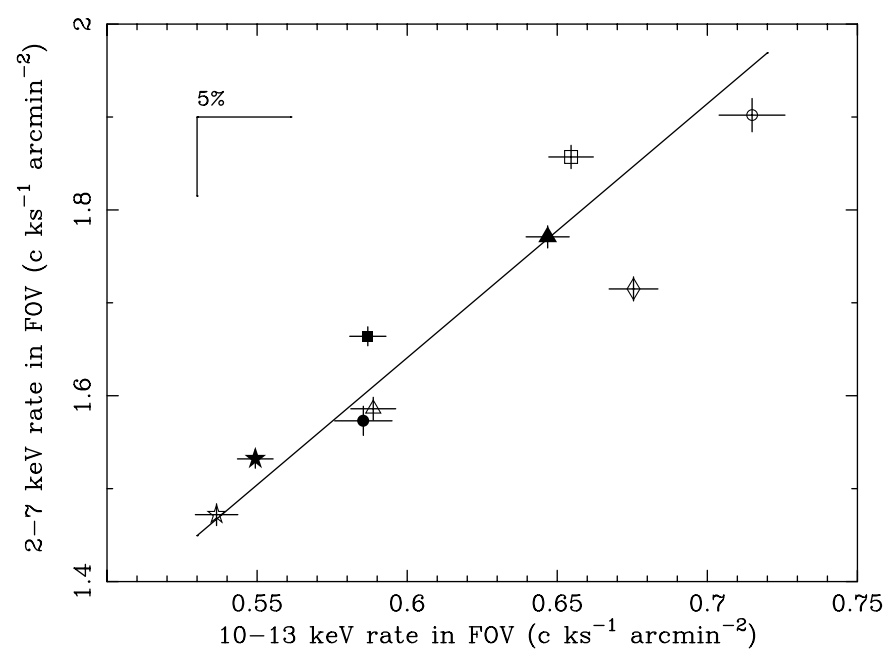

Fig. 12. Correlation between the $10.0-12.0 \mathrm{keV}$ count rate and the $2.0-7.0 \mathrm{keV}$ count rate. The correlation coefficient is 0.929 . The meaning of the symbols are summarized in Table 1 . The solid line corresponds to the best fit regression line.

the improvement from $8 \%$ to $2 \%$ except in the central 4 arcmin radius region, where the contribution from the cosmic X-ray background is the maximum.

\section{Comparison with ASCA and Chandra}

In this section, we compare the spectra of the background of different X-ray CCDs onboard various satellites, which include ASCA/SIS, Chandra/ACIS-I and ACIS-S, XMM/EPIC-pn and EPIC-MOS. We here treat these detectors separately, although ASCA/SIS and XMM/EPIC are equipped with multiple detectors. For ASCA/SIS, we used a blank-sky data base provided by NASA/GSFC, while the Chandra ACIS-S and ACIS-I data used here are taken from blank sky observations compiled by Markevitch (2001). Each spectrum is normalized by the physical size of the CCD, so that they represent the background

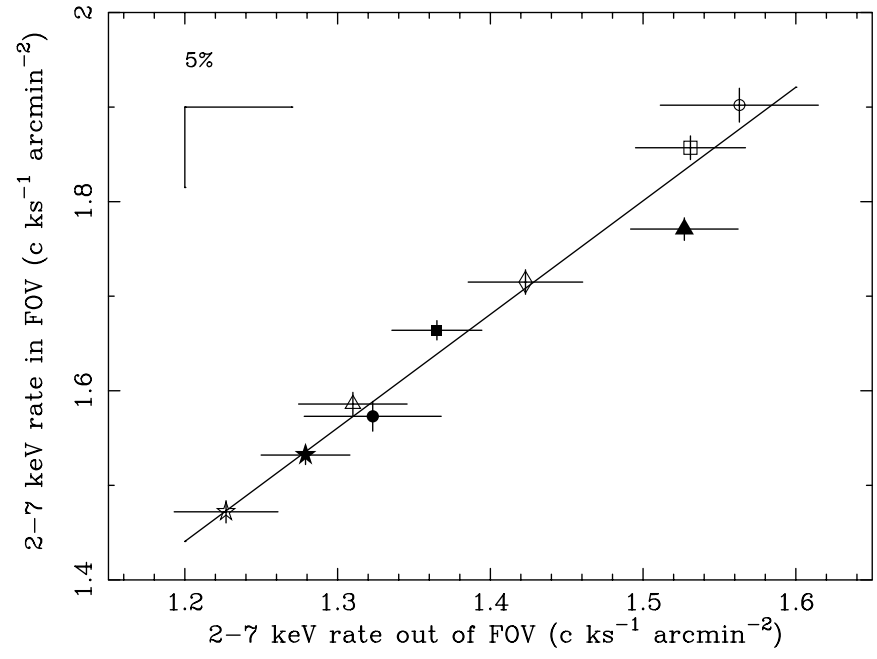

Fig. 13. Correlation between the $2.0-7.0 \mathrm{keV}$ count rate of outside of FOV and that of in FOV. The correlation coefficient is 0.983 . The meaning of the symbols are summarized in Table 1. The solid line corresponds to the best fit regression line.

count rate per unit area on the focal plane. The normalized spectra are shown in Fig. 15. As these spectra include the CXB component, a direct comparison of the particle event flux is meaningful only above about $5 \mathrm{keV}$. The difference of the background count rates among different instruments should be explained by the cosmic-ray-particle flux depending on the satellite orbit, and the sensitivity of the CCDs, which is governed by the thickness of the depletion layer and other structure (e.g. front-illuminated or back-illuminated).

In Fig. 16, each spectrum is normalized by the effective area of the X-ray telescope plus CCD at its focus and by the solid angle of the FOV. Thus, it represents a surface brightness of the background, and gives a measure of sensitivity to diffuse objects. Although the $\mathrm{S} / \mathrm{N}$ ratio also depends on the angular size of X-ray emission and the PSF of the telescope, we here compare the sensitivity to very extended objects like nearby clusters or SNRs. Note that the XMM/EPIC-pn has 10 times larger effective area but 2 times longer focal length than the ASCA/SIS, which reduces background count rate by a factor of $\left(2^{2} / 10\right)=0.4$ for the same count rates per unit area on the focal plane. Since the particle background count rate for XMM is about 5 times larger than that of ASCA/SIS, the ASCA/SIS is still the most sensitive for faint diffuse sources among those CCDs.

\section{Summary and discussion}

We investigated the properties of the EPIC-pn background. A typical light curve of an observation shows violent variabilities due to the soft protons, while, after eliminating such high count rate periods, the count rate is found to be rather stable. The background energy spectrum is consist of high energy particle events (continuum and characteristic emission lines) dominating above $5 \mathrm{keV}$, the cosmic X-ray background dominating in the $0.4-5.0 \mathrm{keV}$ band, and the non-X-ray noise contributing only below $0.4 \mathrm{keV}$. Background count rate from April 2000 to May 2001 is found to be stable within $8 \%$ depending on 

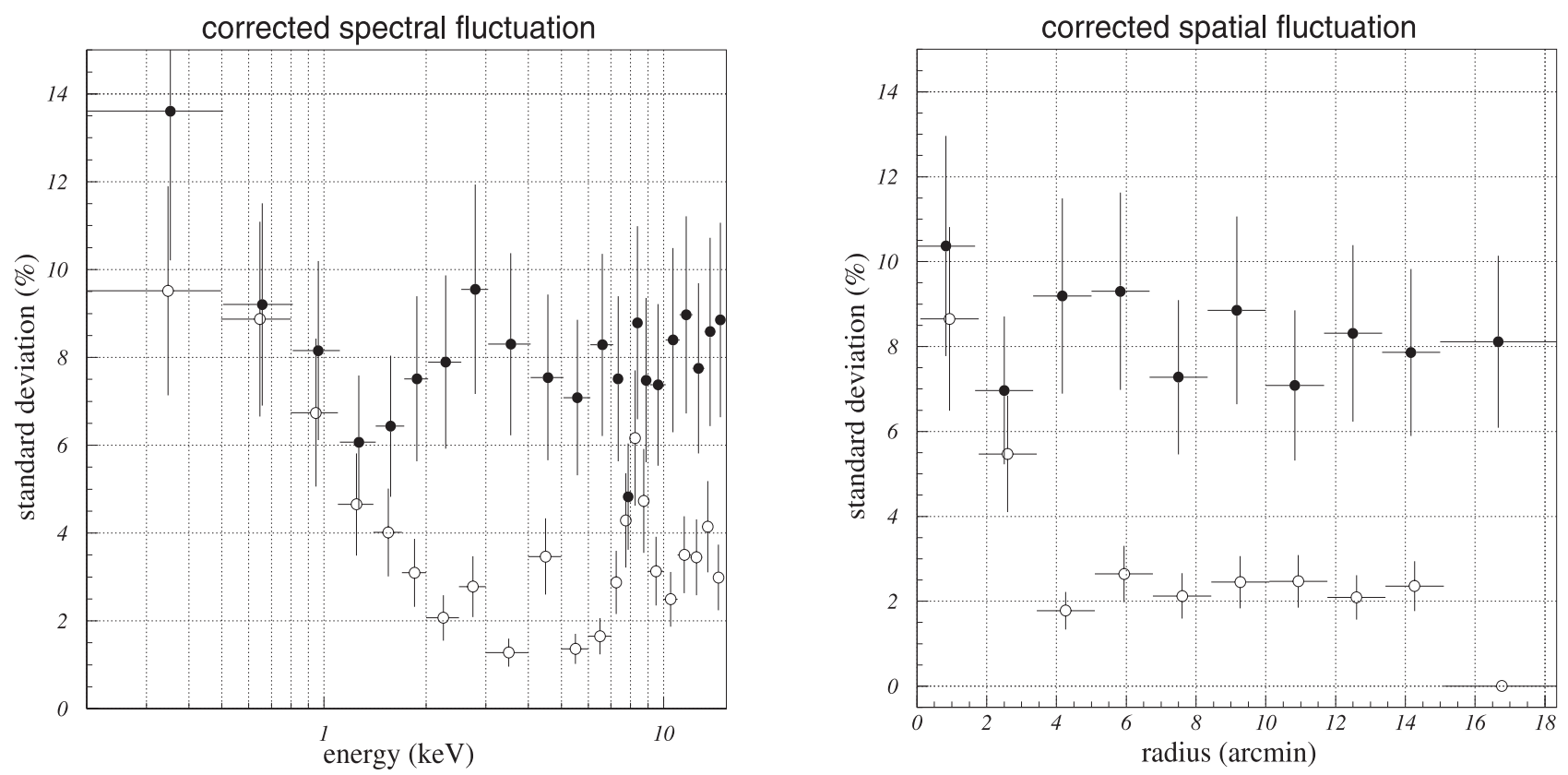

Fig. 14. Standard deviation of corrected spectra (left $)$ and 2.0-7.0 keV spatial distributions (right). Filled circles and open circles represent data before correction and after correction, respectively.

ASCA, Chandra, XMM-Newton background spectra

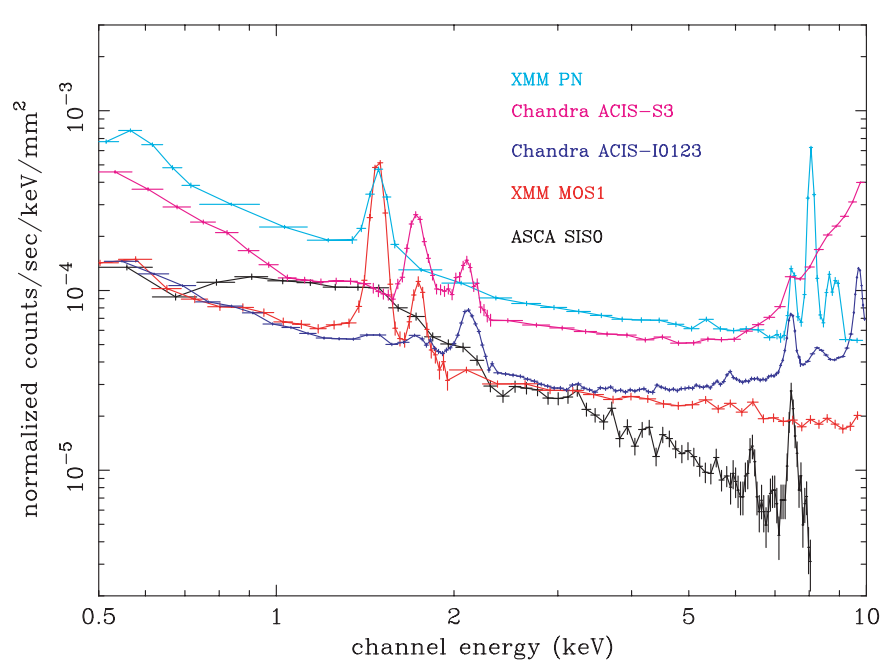

Fig. 15. XMM, Chandra, and ASCA background spectra normalized by $\mathrm{CCD}$ area.

energy, but it gradually decreased by $20 \%$ by the end of January 2001. In addition to this long term trend, background count rate of an observation could fluctuate as large as 50\% from the average. We found that the events detected in the out of field of view well represent the particle origin background in the field of view as well, which can be utilized for modeling the background. For an actual modeling of the background, we also need take into account the variation of the soft CXB below $2 \mathrm{keV}$ from sky to sky (see e.g. Snowden et al. 1997).

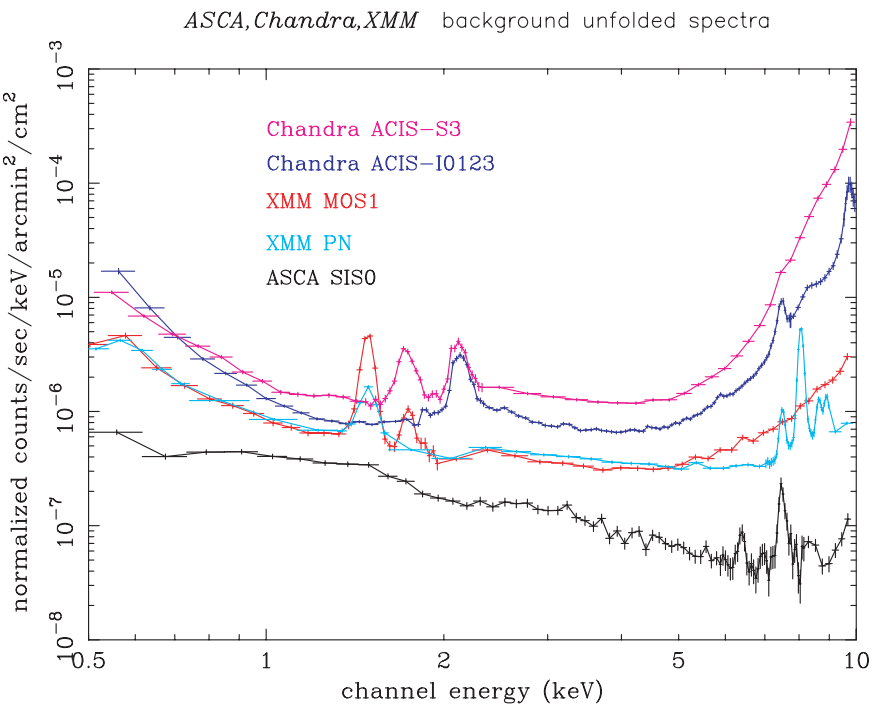

Fig. 16. XMM, Chandra, and ASCA background spectra normalized by effective area.

Acknowledgements. This work is based on observations obtained with XMM-Newton, an ESA science mission with instruments and contributions directly funded by ESA Member States and the USA (NASA). We are grateful to $\mathrm{H}$. Böhringer and Y. Tanaka for useful comments. This work is supported by the GermanJapan collaboration program funded by the Japan Society for the Promotion of Science and Max-Planck-Institut für extraterrestrische Physik. 


\section{References}

Briel, U. G., Henry, J. P., Lumb, D. H., et al. 2001, A\&A, 365, L60 Freyberg, M. J., Pfeffermann, E, \& Briel, U. G. 2002a, in Symp. New Visions of the X-ray Universe in the XMM-Newton and Chandra Era (ESA SP-488; Noordwijk: ESA)

Freyberg, M. J., Briel, U. G., Dennerl, K., et al. 2002b, in Symp. New Visions of the X-ray Universe in the XMM-Newton and Chandra Era (ESA SP-488; Noordwijk: ESA)

Gendreau, K. C. 1995a, Ph.D. Thesis, MIT

Gendreau, K. C., Mushotzky R., Fabian A. C., et al. 1995b, PASJ, 47, L5

Jansen, F., Lumb, D., Altieri, B., et al. 2001, A\&A, 365, L1
Lumb, D. 2001, XMM-SOC-CAL-TN-0016

Markevitch, M. 2001, http://asc.harvard.edu/cal/Links/ Acis/acis/Cal_prods/bkgrnd/current/index.html Marty, P. B., Kneib, J., Sadat, R., Ebeling, H., \& Smail, I. 2003, Proc. SPIE, 4851, 208

Prigozhin, G., Kissel, S., Bautz, M., et al. 2000, Proc. SPIE, 4012, 720 Snowden, S. L., Egger, R., Freyberg, M. J., et al. 1997, ApJ, 485, 125 Strüder, L., Briel, U., Dennerl, K., et al. 2001, A\&A, 365, L18 Tanaka, Y., \& Bleeker, J. A. M. 1977, Space Sci. Rev., 20, 815 Tanaka, Y., Inoue, H., \& Holt, S. S. 1994, PASJ, 46, L37

Ueda, Y. 1996, Ph.D. Thesis, The University of Tokyo

Weisskopf, M. C., Tananbaum, H. D., Van Speybroeck, L. P., \& O’Dell, S. L. 2000, Proc. SPIE, 4012, 2 